\title{
POSITION BASED INDOOR AND OUTDOOR NAVIGATION SYSTEM FOR VISUALLY IMPAIRED WITH MIND MAPPING TECHNIQUES
}

\begin{tabular}{|l|l|l|}
\hline Pasam Kiran Teja & Vishwanath S Kaddi & Rakhi S \\
Student, Atria Institute of Technology & Student, Atria Institute of Technology & Faculty, Atria Institute of Technology \\
$1^{\text {st }}$ main, AG’s Colony, Anandnagar, & $1^{\text {st }}$ main, AG's Colony, Anandnagar, & $1^{\text {st }}$ main, AG's Colony, Anandnagar, \\
Bangalore-560024 & Bangalore-560024 & Bangalore-560024 \\
pasamkiranteja93@gmail.com & 93vishu@gmail.com & rakhinit@ rediffmail.com \\
\hline
\end{tabular}

\begin{abstract}
The ability to navigate from place to place is an integral part of daily life.Indoor navigation is an important need of visually impaired which a GPS(Global Positioning System) cannot provide using the existing technologies. In modern days GPS based navigation has gained a lot of popularity. GPS provides information about a person's location almost anywhere in the world when navigating outdoors. However GPS based navigation has some notable limitations.Since GPS signals are line of sight they are often disrupted when the user is navigating under dense foliage or between tall buildings. This paper proposes a cheap and affordable solution of navigation for visually impaired without GPS overcoming the disadvantages and maintaining all its major features.Both indoor and outdoor navigation have been facilitated by a common set of algorithms and hardware.It uses a novel approach in constructing a map using raw position values of the person and then using this map as a knowledge base in navigating the blind person to the destination.
\end{abstract}

\section{Keywords- navigation,tracking,GPS, map, RFID}

\section{Introduction}

Navigation can be divided into two aspects: Indoor and Outdoor Navigation. Several technologies have been implemented to provide either indoor or outdoor navigation. So far, no single technology has been implemented to provide both indoor and outdoor navigation facility using a single set of hardware. There has always been a tradeoff between the ability of facilitating indoor navigation and that of outdoor navigation. Hardware suitable for indoor could not be suitable for outdoor navigation and vice versa.GPS technology is comparatively the best when it comes to outdoor navigation, while it is not suitable for indoor. Talking signs or arrows were used to direct a blind person from one room to another indoor and these signs are analyzed using image processing but this would require the entire indoor environment be filled with arrows and hence is not feasible. RFID based indoor navigation also would require installation of RFID's on all the locations of interest. Hence there is a need for a cheap and affordable technology which provides the facility of both indoor and outdoor navigation to the blind in a single hardware setup. This project is the first step towards that objective where the navigation information is being given to the blind in the tactile form(through vibrator motor) in order to cut down on the cost [1].

In addition to this, our project also proves the concept of how one can navigate in an outdoor environment without using GPS.

GPS can help a person navigate to a park but cannot help him navigate within the park.It can help the blind person navigate to a hospital but cannot help him further in moving from one room to another.Hence the limitations of GPS are solved in this project where the map is first constructed using a mathematical algorithm proposed by us in this paper and then the map is used as a knowledge base in navigating the blind to the destination.

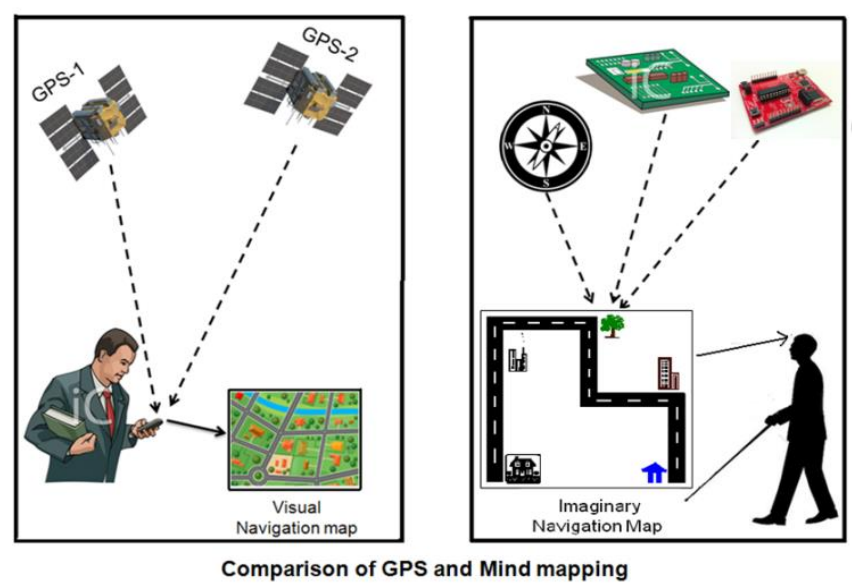

Fig 1. Comparison of GPS and Mind mapping 
The idea is to use a learning based approach for navigation purpose where the system will be in learning mode during the first time journey to the destination. This requires external guidance but only for the first time journey. The guide takes the blind person to the destination and meanwhile learning mode is activated. During the learning mode the position of the blind person walking on the road is tracked using the direction information and number of steps in that direction. These position values are then used to determine the direction of the road in which the blind person is walking. The algorithm used for map construction will be explained in later sections of this paper. The map created is then used as a knowledge base to navigate the blind person to the destination in all the subsequent journeys.

\section{Organization of the paper:}

The paper is organized as follows:Section II describes the learning mode algorithm and implementation.Section III contains the description of Navigation mode algorithm and implementation.Section IV consists of test results andoverall performance analysis of the prototype.Section $\mathrm{V}$ includes the conclusions the future prospects.

\section{II.LEARNING MODE}

Learning mode is the process of creating a map using the position values of the blind person during the journey from source to destination.

\section{A.Tracking the position:}

Position tracking is achieved by combining the direction information along with the number of steps of the blind person. A push button switch is used to count the number of the steps and is mounted at the bottom of the shoe or footwear worn by the blind person as shown below in Figure 3.The figure shows how the position information is obtained by combining the compass output with the number of steps obtained from the switch. The switch is connected as an interrupt to the microcontroller MSP430f5529. Each time the shoe switch interrupt occurs, a count value is incremented yielding the number of steps. The compass reading is combined with the number of steps to get the position value of the blind person. The distance per step is assumed constant. These position values are then used to create the map[1].

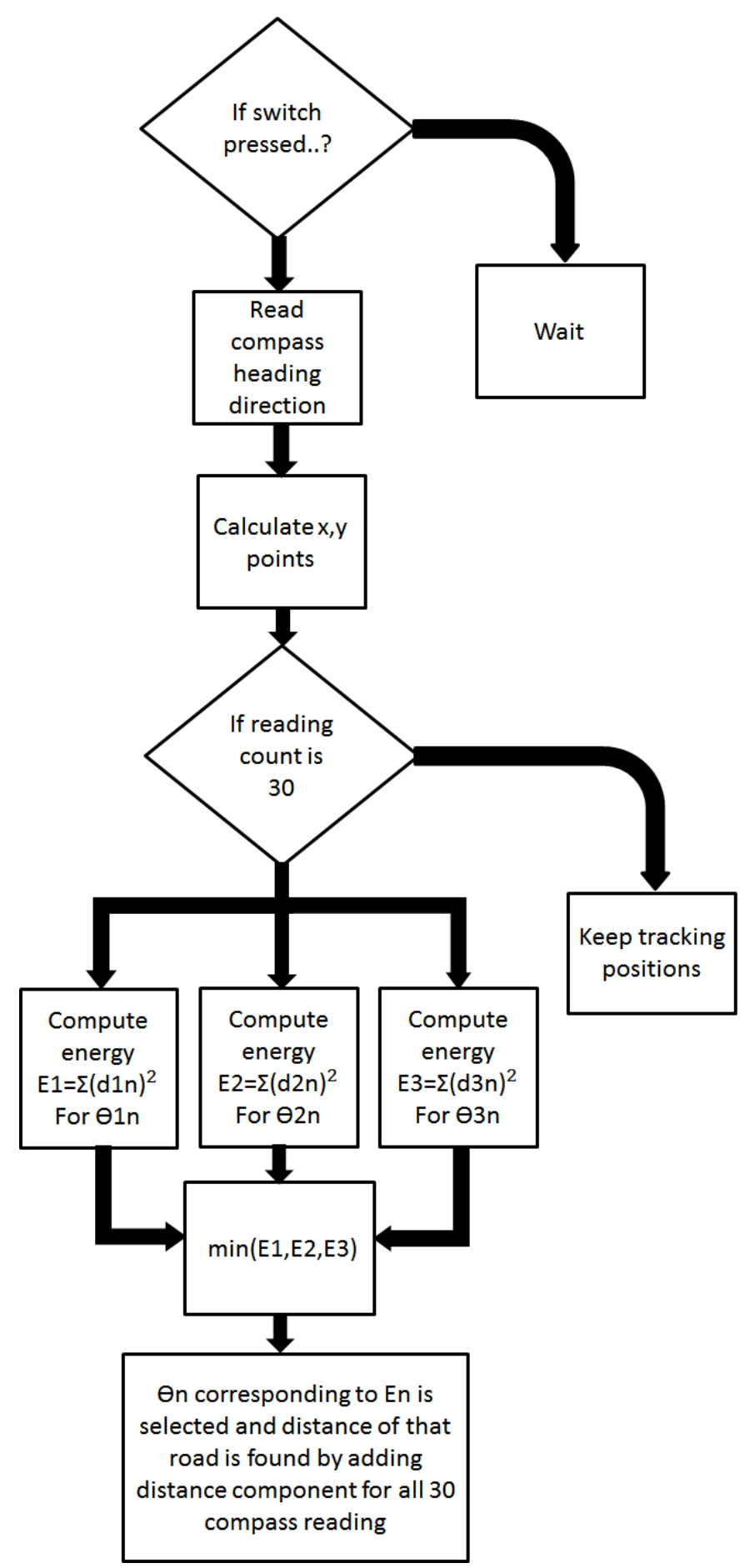

Fig 2. Flowchart of learning mode 


\section{ELK}

Asia Pacific Journals

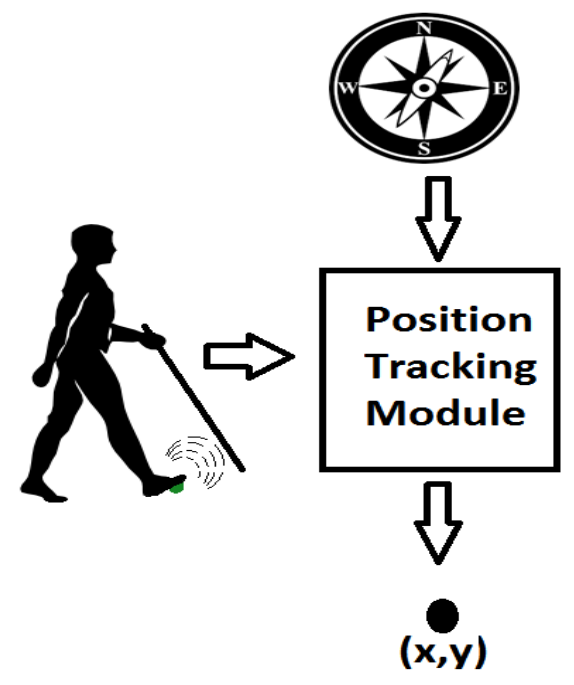

Fig 3. Obtaining direction and distance

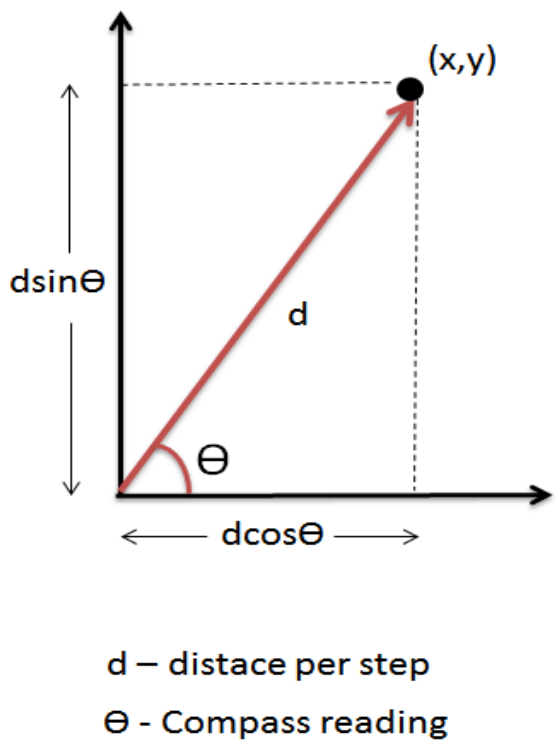

Fig 4.

\section{B. Map Creation}

The task of creating a map is not straightforward.It involves determining the direction and distance of each road in the map.The approach is to consider a set of 30 position values each and then applying a set of algorithms. The first step is to determine the quadrant to which the road direction belongs to.It is explained using the figure shown below.

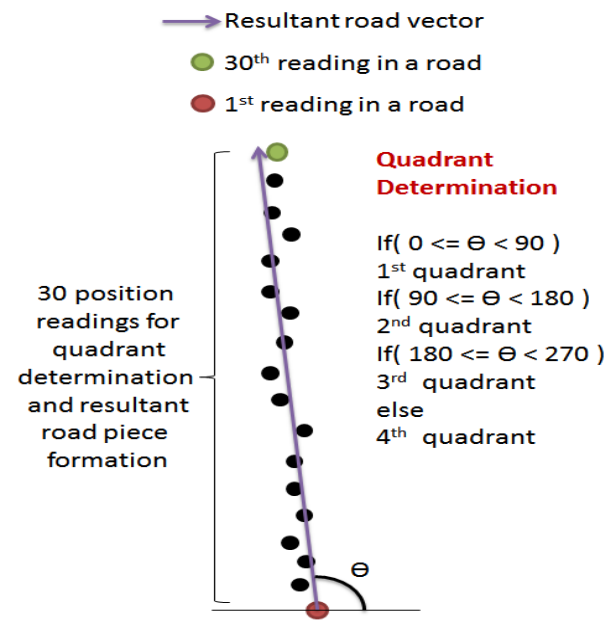

Fig 5. Quadrant determination

The purpose of quadrant determination is to filter out the most probable road directions from a set of predefined road directions. Suppose the controller is having eight possible road directions as its predefined knowledge base i.e. $\left\{0^{\circ}, 45^{\circ}, 90^{\circ}, 135^{\circ}, 180^{\circ}, 225^{\circ}, 270^{\circ}, 315^{\circ}, 360^{\circ}\right\}$.Quadrant determination extracts only three angles from a set of eight possible angles.This reduces the number of computations.For example,if it is the first quadrant the most probable directions are $0,45^{\circ}, 90^{\circ}$. The way in which the quadrant is determined is to compute inverse tangent of the slope between the initial and final point as shown in the above figure. Once the quadrant is determined the next step is to apply the minimum energy algorithm to each of the most probable road directions.

\section{C.Minimum Energy Algorithm}

The heart of map creation is the minimum energy algorithm that we use to determine the exact direction of the road.One cannot determine the direction of the road directly by taking into consideration the initial and final point of the set of 30 position values.Every position value is important and must be considered in determining the direction of the road. There is an ambiguity in road direction determination if we consider only the initial and final point.For example,if the inverse tangent of the slope between the initial and the final pointis $45^{\circ}$ it does not always mean that the direction of the road is $45^{\circ}$.It may even be a straight vertical road of $90^{\circ}$ direction. This ambiguity arises because the road is two dimensional and hence the person can land up in a point that is $45^{\circ}$ inclined with the initial point even though the net direction or the actual direction of the road is $90^{\circ}$. This ambiguity is solved by using minimum energy algorithm[2]. 
The idea behind using minimum energy algorithm is that the direction of the road is that along which the energy of oscillation of all the position values in the set of 30 values is minimum. For example, if it is 90 degree road all the position values within the sector of 30 position values oscillate maximum for $0^{\circ}$, then for $45^{\circ}$, then minimum oscillation for $90^{\circ}$ as shown below.

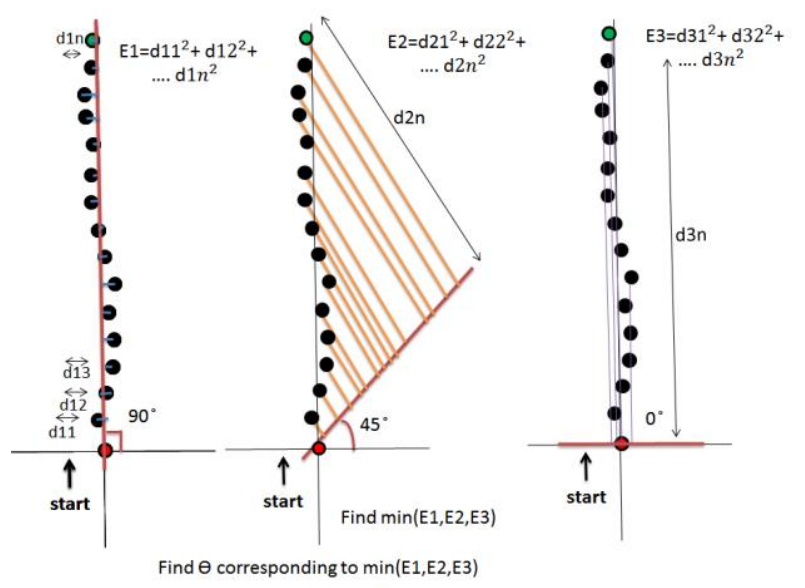

Fig 6. Minimum Energy Algorithm Demonstration

To determine which direction has minimum energy of oscillation of position values, we first need to determine the energy of each direction. Now the question is how to calculate this energy? To calculate the energy we need the perpendicular distance to each position from the central axis which is abbreviated as ' $d$ '. The sum of squares of these $d$ values over the entire set of 30 position values is the energy of that direction. The direction with least energy is the direction of the road.The perpendicular distance ' $d$ ' is determined using the following mathematical calculations [5].The co-ordinates of initial point and final point of the resultant road piece is known which is assumed as $\left(\mathrm{X}_{0}, \mathrm{Y}_{0}\right)$ and $\left(\mathrm{X}_{\mathrm{F}}, \mathrm{Y}_{\mathrm{F}}\right)$ respectively. And also let us assume ' $k$ 'th point with co-ordinate $\left(X_{K}, Y_{K}\right)$ whose perpendicular distance from the resultant road piece has to be found. The length of resultant road is $36 \mathrm{~m}$ for 30 readings of $1.2 \mathrm{~m}$ step length each.So from fig 7 , the lengths $\mathrm{r} 1$ and $\mathrm{r} 2$ can be found from co-ordinates of initial and final point. So there are two unknowns $y$ and $d$ from the figure. By applying Pythagoras theorem to two Right angled triangles, we get two equations as shown below.

Thus the perpendicular distance ' $d$ 'is obtained for particular point ( $\mathrm{k}$ in this occasion) by solving these two equations and process is repeated for remaining 30 position value points

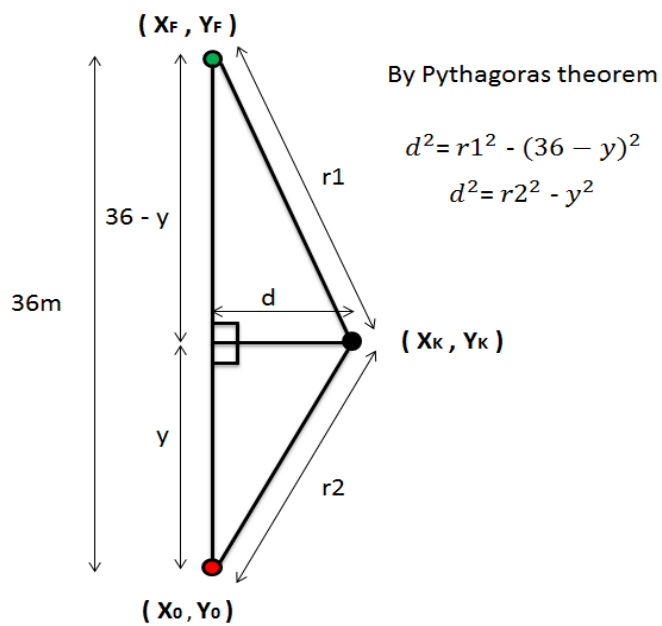

Fig 7. Perpendicular distance 'd' determination

which are further used to find the minimum energies for different road pieces. Now that the direction of the road is determined the further task is to determine the length of the road. The length of the road is either the horizontal or vertical component or the component across 45 or 135 or 225 or 315 of the distance between the initial and the final position value determined using distance formula [6].

\section{$($ XDIST,YDIST $)=(36 \cos \theta, 36 \sin \theta)$}

where 36 (meters) is the length of road

$\theta$ is the resultant angle of road obtained by minimum energy algorithm.In this way road pieces are generated and the map is formed as an array of road pieces and is stored as a knowledge base. This knowledge base is used to navigate the blind person in navigation mode.

\section{Implementation of Learning Mode}

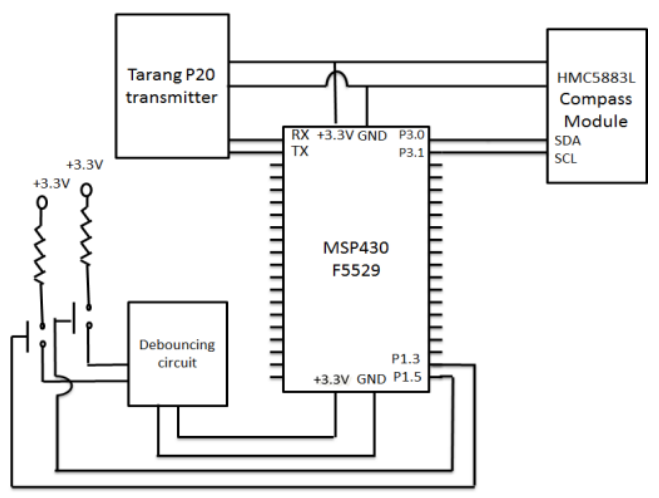

Fig 8. Learning mode Circuit 
It consists of MSP430F5529 microcontroller as the core with the push button switch (mounted under the shoe) connected to the pin P1.5 of the microcontroller as an interrupt. If the push button switch is connected directly to the microcontroller, the interrupt service routine may get executed multiple times due to bouncing effect of the push button switch. Therefore the switch is connected to the microcontroller through the debouncing circuit. The de-bouncing circuit consists of an RC circuit which acts as a low pass filter and slows down the high frequency ripples caused due to bouncing effect of the switch. The output of the capacitor of the RC circuit is then converted into pure digital pulses by a Schmitt trigger IC SL74AC14N(Texas instruments). As a result of de-bouncing the interrupt service routine gets executed only once when the switch is pressed [4].

The circuit also consists of 3 -axis digital compass module HMC5883L connected to the I2C pins (P3.0, P3.1) of the microcontroller. The compass gives the heading information which when combined with number of steps given by the switch output yields the position information of the blind person.An additional push button switch is used to start and stop the learning mode and is connected to P1.3 pin of the microcontroller.Since, MSP430 operates at $5 \mathrm{~V}$ supply and a $9 \mathrm{~V}$ battery has been used, a regulator circuit is required to step down the voltage. For this purpose we use IC 7805 as a voltage regulator.For presentation purpose we have used a wireless TARANG P20 module to transmit the position information and the processed data to the computer. The TARANG module is connected to the microcontroller to the $\mathrm{Rx}$ and $\mathrm{Tx}$ pins of the microcontroller.The supply to the TARANG module is given by $3.3 \mathrm{~V}$ pin of the microcontroller.

\section{NAVIGATION MODE}

In navigation mode, the blind person is guided to the destination independently by the controller without the need of external guide. The map created and stored as a knowledge base in the learning mode is now used in the navigation mode to guide the blind person. To provide the facility of GPS and to cut down on the cost we have used two vibrator motors to inform the person whether to turn left or to turn right when a cross road arrives. As soon as the destination is reached both left and the right vibrator motor vibrate at the same time. To provide mobility to the blind person in presence of obstacles we use HCSR04 ultrasonic module connected to Arduino Uno microcontroller which acts as a standalone obstacle detection and avoidance unit. This reduces the burden of the MSP430F5529 in managing all the tasks and allows it to focus only on learning mode map creation and navigation mode guiding system. Another vibrator motor is used to act like obstacle avoidance step. The approach in navigation mode is to calculate the distance of progress of blind person in each road and to warn the person as soon as the distance left in each rod is less than 1.5 meters. The distance left at any given time is the difference between the actual length of the road and the distance progressed at that time in that road [8].

\section{A. Implementation of Navigation Mode}

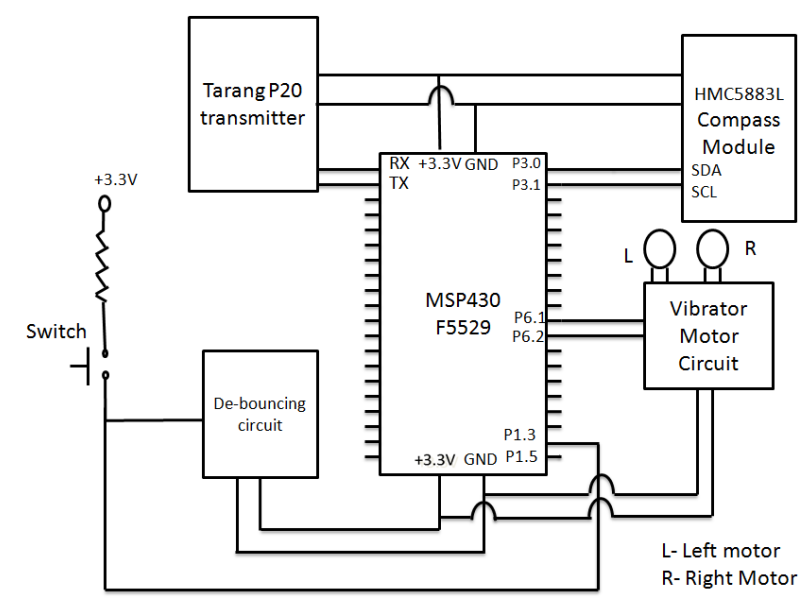

Fig 9. Navigation mode circuit

It consists of the MSP430F5529(Texas instruments) microcontroller as the core. The 3-axis digital compass HMC5883L is connected to the controller via I2C pins. The shoe switch is connected to the microcontroller interrupt pin P1.5 through an RC de-bouncing circuit.To guide the blind person we need two vibrator motors namely left and right vibrator motor. Since the output digital high voltage level of MSP430F5529 is only 3.3V and for significant recognizable vibration the voltage must be $5 \mathrm{~V}$, we use a vibrator driver circuit which is basically an npn transistor BC547 the base of which isconnected to the vibrator output pin of the microcontroller (P6.2, P6.1). The collector of the transistor is connected to the $5 \mathrm{v}$ supply and the emitter to the vibrator motor. As soon as the transistor turns on, the $5 \mathrm{~V}$ supply is directly connected to the vibrator circuit.The switch on and off action of the transistor is controlled by the vibrator output pin of the microcontroller.The obstacle detection and avoidance system consists of HCSR04 ultrasonic sensor connected to the Arduino Uno.The obstacle vibrator vibrates whenever an obstacle is within the specified range. 


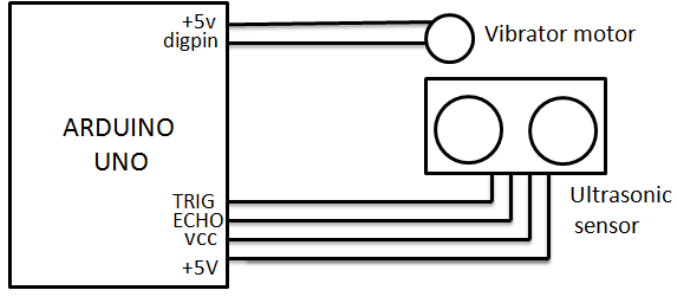

Fig 10. Ultrasonic sensor with Arduino uno

\section{RESULTS}

The starting point of journey in the learning mode is the origin and the position of blind person is tracked from that point only. The direction in which the person starts his journey with the help of the guide is always taken as $90^{\circ}$ in the learning mode and he is supposed to start his independent journey in navigation mode in that direction only. The position tracked at every step of blind person is indicated by red colored plus symbol as shown in figure below.

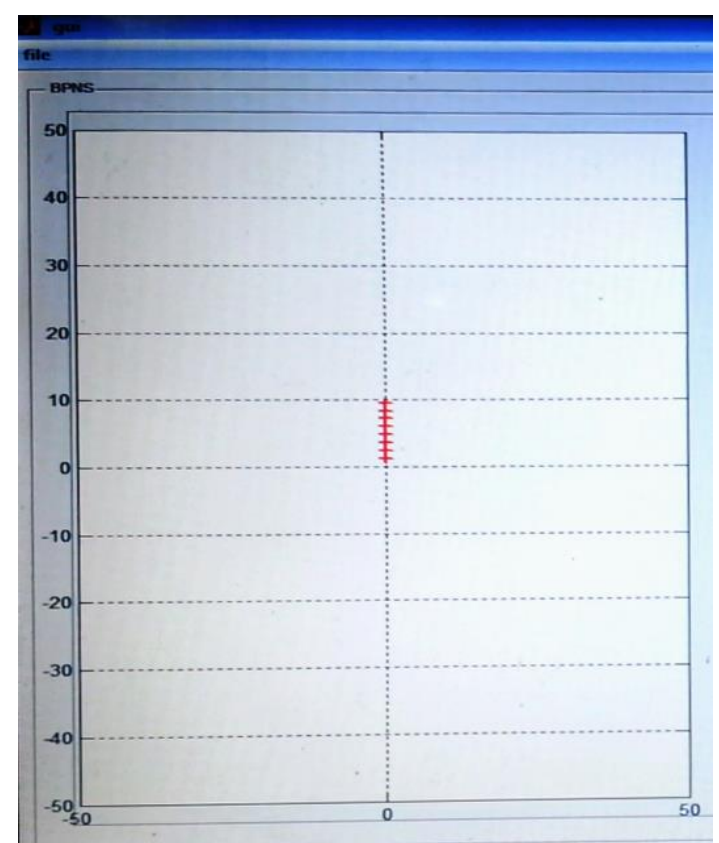

Fig 11. Learning mode map1

The position of the blind person is tracked and dispayed continuously. But after every such 30 readings, the microprocessor takes those readings and applies the algorithm which has been discussed in the earlier part of the paper to obtain the resultant road piece which is indicated with the blue thin line(which here is scaled line of $36 \mathrm{~m}$ ) shown in the fig. 12

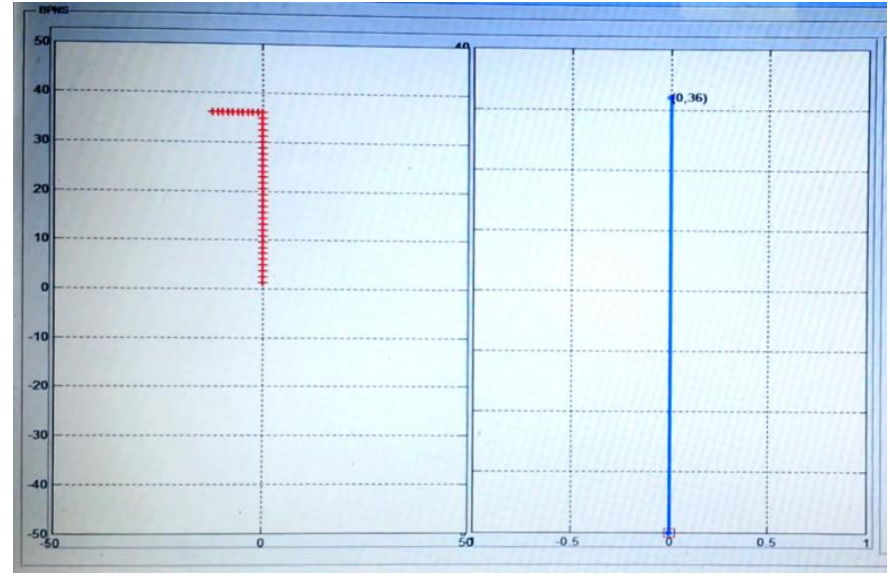

Fig 12. Learning mode map2

The final map created is shown below along with all the points tracked by the blind person. The person has travelled around $36 \mathrm{~m}$ in $90^{\circ}$ and then has taken left cross which is shown by $180^{\circ}$ line. When the destination is reached, the guide asks the blind person to press the button which ends the learning mode. As a result of which the position tracking is stopped and final map is obtained.

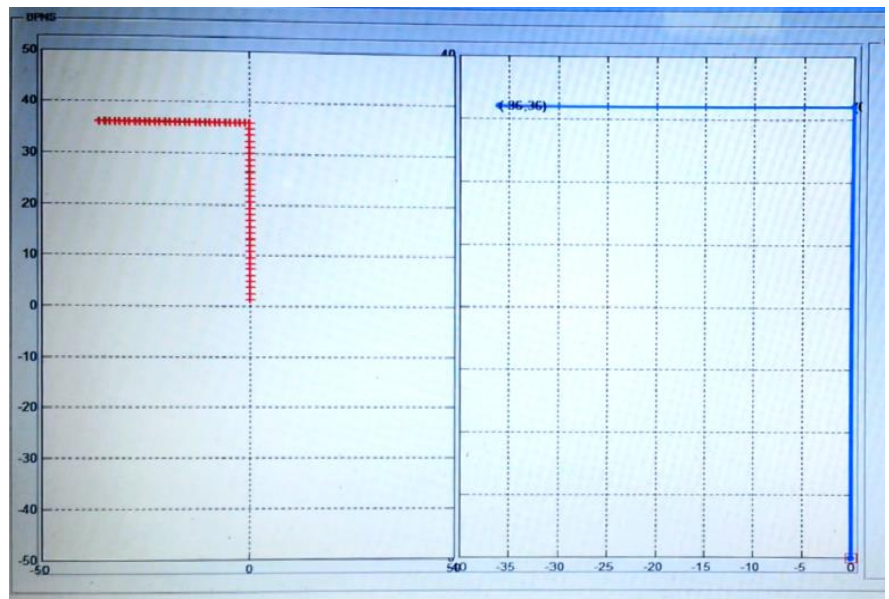

Fig 13. Learning mode map3

The map created in learning mode with its resultant distance and direction is taken as a reference for navigation mode and the same map is indicated with very thick blue line as shown in fig. 14. As the person starts his journey in navigation mode, his position is again tracked by the controller with respect to the map generated. Now the position at every instant in navigation mode is shown with the green plus symbol on the thick blue line. 


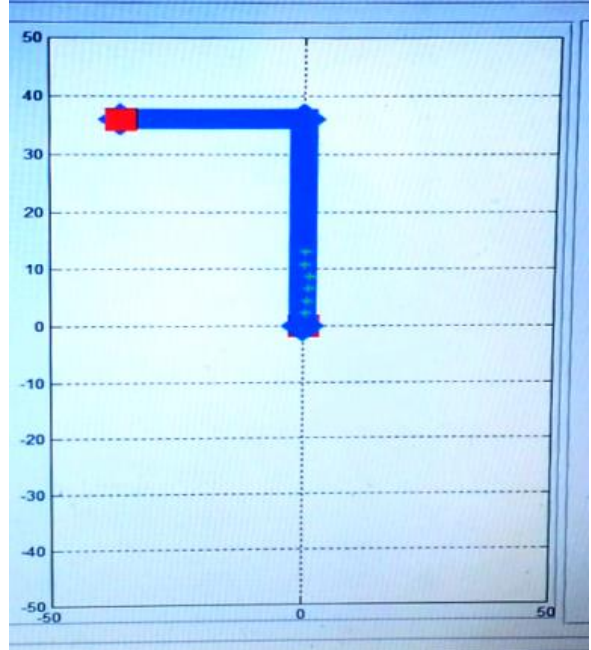

Fig 14. Navigation mode map1

When the person reaches the end of the road he is guided to take the left or right turn with the help of two vibrators mounted on either side of the belt. Here when person comes to end of $90^{\circ}$ road, the left vibrator vibrates instantly to help him to take the left turn.

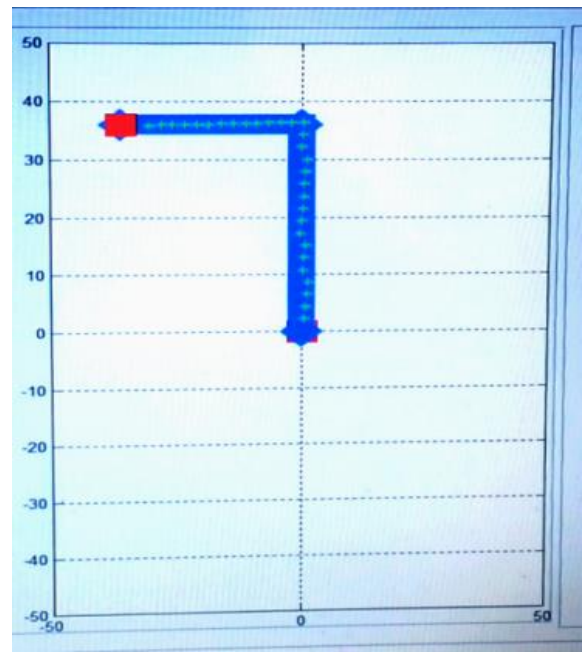

Fig 15. Navigation mode map2

The thick red dot is the terminal point of the map. When the green colored plus symbol touches that dot, it indicates that the person has reached his destination in Navigation mode. This is indicated by both left and right vibrators vibrating simultaneously. And also the Arduino Uno board with an Ultrasonic sensor and separate vibrator motor helps the blindperson to move independently in Navigation mode by avoiding the obstacles.
The performance analysis for this application is very important as this is a hard real time system application. The main factors that need to be considered in this analysis are the Accuracy of distance and direction, Time response of the system, Size and ease of usage, and the cost. The system uses a switch which gives the step count. And the step length is assumed to be $1.2 \mathrm{~m}$ which is average step distance covered by the person. But this may vary slightly for a tall person or a person with short stature. So, one needs to choose the step length accordingly. There is also an encouragement for measuring the variation in step length of same person using tilt sensors like accelerometer, gyroscope etc. But a person walking with variable step length is not a usual scenario.The system has a very good time response as it updates the position of person on pressing of shoe switch instantly and it gives the resultant road information immediately after every $30^{\text {th }}$ reading. The obstacle avoiding system uses a simple ultrasonic sensor HCSR04 which has detecting range of $2 \mathrm{~m}$. A long range ultrasonic sensor can be used to detect the obstacle in advance and to give enough time for the blind person to take required decisions. But long range ultrasonic sensors are much costly comparatively.

The system is made very user friendly. It's a relatively small box attached to a thin belt. All the hardware circuitries with rechargeable batteries are fitted inside this small box and compass, ultrasonic sensor with vibrators is attached at definite positions on the belt. The belt-box arrangement is shown in figure 16. The learning mode start/stop button is on one the top side of the box which is made very easy for blind person to locate and access. And the belt can also be worn easily.The application is very much affordable for the blind person. The prototype has been implemented with the hardware cost being less than INR 2000.

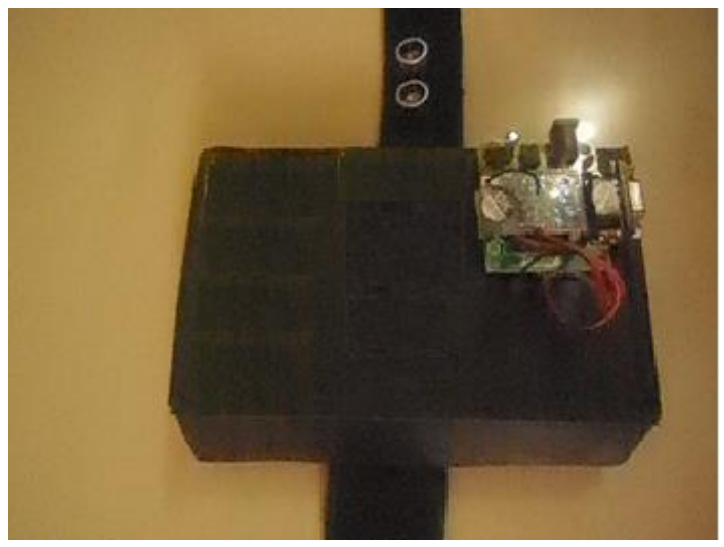

Fig. 16. Box-Belt Arrangement 


\section{CONCLUSION\& FUTURE SCOPE}

This paper proves that one can navigate a robot or a visually impaired person both in indoor and outdoor environment using a common set of hardware. The cost of implementation is less compared to GPS systems. Inability to navigate in indoor environment, network coverage issues, effect of atmospheric conditions(such as dense fog) on navigation accuracy are avoided in this system.

When the number of destinations is too large to store in onboard memory, a main frame computer system can beinstalled in the house of the blind person, can be used to store the huge database of maps to multiple destination. One can also store different routes to the same destination.

\section{REFERENCES}

[1] Dimitrios Dakopoulos and Nikolaos G. Bourbakis, "Wearable Obstacle Avoidance Electronic Travel Aids for Blind: A Survey", IEEE Transactions on Systems, man, and cybernetics- part c: application and reviews, volume 40, January 2010.

[2] Mounir Bousbia-Salah , Abdelghani Redjati, Mohamed Fezari, Maamar Bettayeb, "An Ultrasonic Navigation System For Blind People", IEEE International Conference on Signal Processing and Communications (ICSPC 2007),Dubai,2427November2007,pp. 1003-1006.

[3] Rusen Oktem, Elif Aydın, Nergiz Ercil Cagiltay, "An Indoor Navigation Aid Designed for Visually Impaired People”, IEEE, 2014, pp. 2982-2987.

[4] Arjun Sharma, Rahul Patidar, Shubham Mandovara, Ishwar Rathod, "Blind Audio Guidance System", International Journal of Emerging Technology and Advanced Engineering, volume 3, January 2013,pp.17-19.

[5] N.Mahmud, R.K.Saha, R.B. Zafar, M.B.H. Bhuian, and S.S.Sarwar, "Vibration and Voice Operated Navigation System for Visually Impaired Person", 3rd International Conference on Informatics, Electronics \& Vision, 2014. [6] A. Aladren, G. Lopez-Nicolas, Luis Puig, and Josechu J. Guerrero, "Navigation Assistance for the Visually Impaired Using RGB-D Sensor With Range Expansion", 2014 IEEE.

[6]Chaithali.K,dr.Prakash,"Review paper on navigation system for visually impaired", International Journal of Advanced Research in Computer and Communication Engineering Vol. 4, Issue 1, January 2015]

[7] A Remote Guidance System for the Blind

Przemyslaw Baranski, Maciej Polanczyk, Pawel StrumilloAvailable
URL:www.researchgate.net/profile/.../00b7d5285407d7a97d0 00000.pdf

[8]Navigation for the Blind Using GPS along with Portable Camera Based Real Time Monitoring

K.Chandana, G.R.Hemantha Available at URL: http://www.internationaljournalssrg.org/IJECE/2014/Volume1 -Issue8/IJECE-V1I8P109.pdf

[9] A Smart Infrared Microcontroller-Based Blind Guidance SystemAmjed S. Al-Fahoum, Heba B. Al-Hmoud, and Ausaila A. Al-Fraihat

[10] Larisa Dunai,",Design, modeling and analysis of objectlocalization through acoustical signalsfor cognitive electronic travel aid forblind people.

Department of Graphics EngineeringSchool of Design Engineering Author:

[11]MSP-EXP430F5529LP LaunchPad ${ }^{\mathrm{TM}}$ Development Kit User's Guide www.ti.com/lit/pdf/slau533 